\title{
Cognitive and mood improvements following acute supplementation with purple grape juice in healthy young adults
}

\author{
C. F. Haskell-Ramsay ${ }^{1}$ R. C. Stuart ${ }^{1}$ E. J. Okello ${ }^{2}$ A. W. Watson ${ }^{2}$
}

Received: 9 August 2016 / Accepted: 11 April 2017 / Published online: 20 April 2017

(C) The Author(s) 2017. This article is an open access publication

\begin{abstract}
Purpose Berry-derived phenolic compounds found in grapes have been associated with a number of health benefits, including the augmentation of human brain function and cognition. Previous intervention studies of Concord grape juice have demonstrated improvement to memory and driving ability following 3- to 4-month supplementation in middle-aged and older adults. However, no studies to date have demonstrated acute cognitive benefits of grape juice, and investigation of these effects in young adults is lacking. Methods This randomised, placebo-controlled, doubleblind, counterbalanced-crossover study, assessed the effects of $230 \mathrm{ml}$ purple grape juice or sugar-matched control in 20 healthy young adults. Computerised measures of episodic memory, working memory, attention and mood were completed at baseline and following a 20-min absorption period. Results Purple grape juice significantly improved reaction time on a composite attention measure $(p=0.047)$ and increased calm ratings $(p=0.046)$ when compared to placebo. Order effects also indicated an enduring positive effect on pre-dose memory reaction time $(p=0.018)$ and post-dose calm ratings $(p=0.019)$ when purple grape was consumed first.

Conclusions These findings in a small sample of healthy young adults suggest that purple grape juice can acutely
\end{abstract}

The original version of this article was revised.

$\triangle$ C. F. Haskell-Ramsay

crystal.haskell-ramsay@northumbria.ac.uk

1 Brain, Performance and Nutrition Research Centre, Northumbria University, Newcastle upon Tyne NE1 8ST, UK

2 School of Agriculture, Food and Rural Development, Newcastle University, Newcastle upon Tyne NE1 7RU, UK enhance aspects of cognition and mood. No significant effects of juice were observed on memory measures, suggesting that these may be less susceptible to manipulation following acute supplementation in healthy young adults. Potential mechanisms underlying these effects include modulation of cerebral blood flow, glucoregulation and inhibition of monoamine oxidase activity, all of which require further exploration.

Keywords Cognition $\cdot$ Cognitive $\cdot$ Mood $\cdot$ Grape . Phenolic $\cdot$ Polyphenol $\cdot$ Phytochemical

\section{Introduction}

Phenolic compounds are found in varying concentrations in a range of plant-based food sources, such as legumes, fruit, vegetables, herbal extracts, spices, coffee, tea and cocoa. These compounds are grouped into phenolic acids, stilbenes, lignans and flavonoids on the basis of the number of phenol rings; with flavonoids further sub-classified into flavonols, flavones, isoflavones, flavanones, anthocyanidins and flavan-3-ols as a function of their structural complexity [1]. Berry fruits, such as strawberry, blueberry, blackcurrant and grape, represent a rich source of (poly)phenols, which are linked to a number of health benefits including modulation of inflammation [2,3], reductions in risk of cardiovascular disease (see [4] for systematic review), anticancer effects [5], and protection against neurodegenerative diseases [6-8]. In particular, commercially available purple grape juice, containing $65 \%$ Concord grape, was shown to have the highest concentration of (poly)phenols relative to other commercially available fruit juices, as well as a competitively high diversity of individual phenolic compounds $[9,10]$. Concord grapes (Vitis labrusca) contain a unique 
mixture of flavan-3-ols, tartaric esters of hydroxycinnamates, and anthocyanins; the latter of which comprise $46 \%$ of the total phenolic content $[9,10]$.

Recent systematic reviews of epidemiological and intervention studies suggest a beneficial role for berry (poly) phenols in relation to cognition $[11,12]$. These are supported by evidence of a number of potential direct and indirect mechanisms, including their interaction with gut microbiota [13], modulation of neuroinflammation [14], improved cerebrovascular function (reviewed in [15]), modulation of glucoregulation [16] and increased spine density and neurogenesis, particularly in the hippocampus [17]. Flavonoids, at low nanomolar concentrations, also induce synaptic plasticity (for review see [18]) due to modulation of receptor function and gene expression and via interaction with signalling pathways through a number of potential actions including binding to ATP sites, regulation of kinase activity and modulation of transcription factor activation and binding [19, 20]. Specific studies of Concord grape juice (CGJ) are also beginning to emerge, suggesting a positive impact on cognition following shortterm supplementation. In the first of two parallel groups studies in older adults with mild cognitive impairment $(N=12)$, improvements to California Verbal Learning Test (CVLT) list acquisition were observed following 12-week supplementation with $6-9 \mathrm{ml} / \mathrm{kg}$ per day of CGJ (range 444-621 ml/day), when compared to placebo [21]. A similar dosing schedule (range 355-621 ml/day) led to reduced CVLT recognition memory errors and increased activation in the right superior parietal cortex and right middle frontal cortex following 16-week supplementation $(N=21)$ [22]. A recent crossover study demonstrated improvements to visuo-spatial learning and driving performance following a 12 -week intervention with CGJ in 40- to 50-year-old healthy working mothers of preteens. There were also a number of interaction effects with study phase indicating carryover effects of grape juice. Specifically, consumption of CGJ in arm 1 was associated with enduring benefits to verbal recall, executive function and driving ability in the second arm of the study when placebo was consumed [23].

The only study to date to assess cognitive effects following a single serve of purple grape juice [24], failed to demonstrate effects of $10 \mathrm{ml} / \mathrm{kg}$ of CGJ in relation to implicit memory, mood and appetite in 18- to 50-year-old smokers (mean age $26 \pm 7.5$ years). The lack of effects in this study may relate to task selection, as there is no evidence for modulation of implicit memory by phenolic compounds from the available literature. It is also pertinent to note that previous studies in young adults have demonstrated acute cognitive benefits of (poly)phenols following supplementation of cocoa flavan-3-ols [25] and blackcurrant [26]. Effects of (poly)phenols on subjective state have also been observed in healthy young adults following acute supplementation with cocoa [25] and orange juice [27], and it is important that these effects are explored further given the well-established relationship between cognition and mood (e.g. see [28] for review). In light of this, the current randomised, placebo-controlled, double-blind, counterbalanced-crossover study examined the acute cognitive and mood effects of a $230 \mathrm{ml}$ single serving of commercially available purple grape juice in healthy young adults.

\section{Method}

\section{Design}

A randomised, placebo-controlled, double-blind, counterbalanced-crossover design was employed. The study was approved by Northumbria University's Department of Psychology Ethics Committee and was conducted in accordance with the Declaration of Helsinki.

\section{Participants}

Twenty participants (7 males; mean age 21.05 years, SD 0.89 ) were drawn through an opportunity sample within Newcastle upon Tyne and the surrounding areas. Due to previous studies showing acute benefits of (poly)phenols in healthy young adults $[24,25]$, and a lack of research into effects of purple grape juice in this population, the current study assessed effects in a healthy young adult sample. All participants were aged 18-35 years. Female participants were not pregnant or seeking to become pregnant, or lactating. All participants had no pre-existing medical conditions or history of neurological, vascular or psychiatric illness; had no current or historical diagnosis of drug or alcohol abuse; were not currently taking medication (excluding the contraceptive pill) or dietary supplements; did not smoke $>3$ cigarettes per day or consume high levels of caffeine ( $>400 \mathrm{mg} /$ day); did not suffer food allergies or sensitivities; and English was their first language. No payment was made for participation.

\section{Treatment}

Placebo and active treatment were administered as $230 \mathrm{ml}$ drinks. The active treatment consisted of $200 \mathrm{ml}$ Welch's ${ }^{\mathrm{TM}}$ purple grape juice (based upon single serving guidelines at the time of the study) plus $30 \mathrm{ml}$ of Schweppes ${ }^{\mathrm{TM}}$ blackcurrant flavour cordial (containing $14 \mathrm{kcal}$ and $3.1 \mathrm{~g}$ sugar per $100 \mathrm{ml}$ ). Placebo consisted of $200 \mathrm{ml}$ Welch's ${ }^{\mathrm{TM}}$ white grape juice plus $10 \mathrm{ml}$ blackcurrant flavour cordial and $20 \mathrm{ml}$ cold water. Schweppes ${ }^{\mathrm{TM}}$ blackcurrant flavour cordial was added at different volumes to the active and placebo drinks to ensure matched sugar content, this also 
served to mask the flavour of the drinks. On the day of testing, beverages were prepared by an independent third party and administered in an opaque container to prevent any visual differences being detected. In order to confirm the phenolic content of the two drinks, analyses of anthocyanin and total phenolic content were conducted as described below. Composition data can be found in Table 1.

\section{Determination of anthocyanin content}

Total monomeric anthocyanin content was determined by the $\mathrm{pH}$ differential method (AOAC Official Method 2005.2) [29]. Briefly, two dilutions of the fruit juices were prepared, one in a potassium chloride buffer ( $\mathrm{pH} 1.0)$ and the other in a sodium acetate buffer ( $\mathrm{pH} 4.5)$ and absorbance measured at 520 and $700 \mathrm{~nm}$ within 20-min of incubation at room temperature. The anthocyanin pigment concentration was calculated and expressed as $\mathrm{mg} / \mathrm{L}$ cyanidin-3-glucoside equivalents using the following equation:

anthocyanin pigment $=\frac{A \times \mathrm{MW} \times \mathrm{DF} \times 10^{3}}{\varepsilon \times 1}$

where $A=(\mathrm{A} 520-\mathrm{A} 700 \mathrm{~nm}) \mathrm{pH} 1.0-(\mathrm{A} 520-\mathrm{A} 700 \mathrm{~nm})$ $\mathrm{pH} 4.5$; $\mathrm{MW}$ is the molecular weight for cyanidin-3-glucoside (449.2); DF is the dilution factor; 1 is the path length in $\mathrm{cm}$ of the cuvette/spectrophotometer; $\varepsilon=26,900$ is the extinction coefficient, in $\mathrm{L} \times \mathrm{mol}^{-1} \times \mathrm{cm}$ for cyanidin3 -glucoside and $10^{3}$ is the factor for converting from $\mathrm{g}$ to $\mathrm{mg}$.

\section{Determination of total phenolic content}

The phenolic content in the samples was determined using the Folin-Ciocalteu methods in a 96-well microplate according to the procedure by Zhang et al. [30]. A gallic acid standard curve $(3.125-100 \mu \mathrm{g} / \mathrm{ml})$ against a blank at $765 \mathrm{~nm}$ was used to calculate the phenolic content as gallic

Table 1 Nutritional content of drinks

\begin{tabular}{lccrc}
\hline & PGJ & WGJ & Active & Placebo \\
\hline Energy $(\mathrm{kcal})$ & 136 & 138 & 140.2 & 139.4 \\
Sugars $(\mathrm{g})$ & 33 & 33.6 & 33.9 & 33.9 \\
Anthocyanin content $(\mathrm{mg} / \mathrm{l})$ & 136.6 & 0.34 & 138.3 & 1.04 \\
Phenolic content $(\mu \mathrm{g} / \mathrm{ml})^{*}$ & 1681.7 & 100 & 1504.5 & 135.1 \\
\hline
\end{tabular}

$P G J$ pure purple grape juice $(200 \mathrm{ml})$, WGJ pure white grape juice $(200 \mathrm{ml})$, Active active drink administered in the studyPGJ $+30 \mathrm{ml}$ blackcurrant cordial, Placebo WGJ $+10 \mathrm{ml}$ blackcurrant cordial $+20 \mathrm{ml}$ water

\# Total monomeric anthocyanin content expressed as cyanidin-3-glucoside equivalents

* Total phenolic content expressed as gallic acid equivalents acid equivalents (GAE $\mu \mathrm{g} / \mathrm{ml})$. Calculation of content took into account the dilution factor (DF) for each juice.

\section{Cognitive and mood measures}

All cognitive and mood measures were administered using the Computerised Mental Performance Assessment System (COMPASS, Northumbria University, Newcastle upon Tyne, UK), a software platform for the presentation of classic and bespoke computerised cognitive tasks. This platform has previously been shown to be sensitive to a range of nutritional interventions [31-33], including blackcurrant supplementation [26]. The computerised tests were conducted via a laptop computer with all stimuli randomised across participant and assessment. Progress through the tasks was controlled by the participant, with brief instructions given on-screen before the start of each task. Tasks were presented in the same order on each occasion and, with the exception of the paper and pencil tasks (word recall and delayed word recall), responses were made using a response pad. Due to the limited available data regarding the effects of acute consumption of purple grape juice on cognitive performance outcomes, the tasks selected comprised standard psychometric tasks assessing cognitive function across different domains. The entire selection of tasks took approximately 20-min to complete. See Table 2 for order, description and scoring of tasks, as well as domain ascribed (see [34]).

\section{Composite scores}

In order to reduce the number of comparisons, $z$-scores were calculated for all cognitive outcomes by pooling data from all assessments as previously described [33]. The tasks were then divided based on whether they represented a memory task (immediate and delayed word recall, numeric working memory, word recognition, picture recognition) or an attention task (simple reaction time, choice reaction time, digit vigilance). These were then further sub-divided on the basis of whether they measured accuracy (\% accuracy) or reaction time (ms). This created four composite scores:

Memory accuracy $=\left({ }^{\mathrm{z}}\right.$ immediate word recall + ${ }^{\mathrm{z}}$ delayed word recall $+{ }^{\mathrm{z}}$ delayed word recognition + ${ }^{\mathrm{z}}$ delayed picture recognition $+{ }^{\mathrm{z}}$ numeric working memory) $/ 5$.

Memory reaction time $(\mathrm{RT})=\left({ }^{\mathrm{z}} \mathrm{RT}\right.$ delayed word recognition $+{ }^{\mathrm{z}} \mathrm{RT}$ delayed picture recognition $+{ }^{\mathrm{z}} \mathrm{RT}$ numeric working memory) $/ 3$.

Attention accuracy $=\left({ }^{\mathrm{z}}\right.$ choice reaction accuracy $+{ }^{\mathrm{z}}$ digit vigilance accuracy)/2. 
Table 2 Cognitive tasks in order of presentation

\begin{tabular}{ll}
\hline Task & Descriptor \\
\hline Word presentation & $\begin{array}{c}\text { A list of words is displayed on the screen, one } \\
\text { word at a time. In this case, } 15 \text { words were } \\
\text { presented with a display time of } 1 \mathrm{~s} \text { and } \\
\text { inter-stimulus interval of } 1 \mathrm{~s}\end{array}$ \\
Participants are instructed to write down the \\
words that were presented. In this case, $60 \mathrm{~s}$ \\
were given to complete the task \\
A series of photographic images are displayed \\
on the screen, one at a time. In this case, 20 \\
images were presented with a display time \\
of $2 \mathrm{~s}$ and an inter-stimulus interval of $1 \mathrm{~s}$ \\
An upwards pointing arrow is displayed on \\
the screen at irregular intervals. Participants \\
must respond as quickly as they can as soon \\
as they see the arrow appear. In this case, 50 \\
stimuli were presented \\
A fixed number appears on the right of the \\
screen and a series of changing numbers \\
appear on the left of the screen at the rate \\
of 150 per minute. Participants are required \\
to make a response when the number on the \\
left matches the number on the right. In this \\
case the task lasted for 3-min \\
Arrows pointing left and right appear on the \\
screen at irregular intervals. The participant \\
is required to indicate the direction of the \\
arrow as quickly as possible whenever an \\
arrow is displayed, by pressing the cor- \\
responding button. In this case, 50 stimuli \\
were presented
\end{tabular}

Numeric working memory A series of numbers are displayed on the screen, one at a time. Participants are required to memorise these numbers as they appear. Once the series is complete, numbers are displayed one at a time and participants are required to indicate if each number was presented in the previous list or not. In this case, three trials were completed with five target numbers in each trial

Delayed word recall

Participants are instructed to write down the words that were presented to them at the beginning of the assessment. In this case, $60 \mathrm{~s}$ were given to complete the task

$-$

$\%$ of words correctly recalled

Episodic memory

Reaction time (ms)

Attention

Accuracy (\%) and reaction time for the cor-

Working memory

Episodic memory

Episodic memory presentation plus an equal number of decoys are displayed on the screen one at a time. Participants indicate if they remember seeing the word earlier or not

Delayed picture recognition All target pictures shown during Picture presentation plus an equal number of decoys are displayed on the screen one at a time. Participants indicate if they remember seeing the picture earlier or not rect responses (ms)

Accuracy $(\%)$ and reaction time for the correct responses $(\mathrm{ms})$

Accuracy (\%) and reaction time for the correct responses $(\mathrm{ms})$

$\%$ of words correctly recalled

Accuracy (\%) and reaction time for the correct responses (ms)

Attention

Attention

Accuracy $(\%)$ and reaction time for the correct responses $(\mathrm{ms})$
Episodic memory 
Attention $\mathrm{RT}=\left({ }^{\mathrm{z}}\right.$ simple reaction time $+{ }^{\mathrm{z}}$ choice reaction time $+{ }^{\mathrm{z}}$ digit vigilance reaction time)/3.

\section{Subjective mood}

Bond-Lader mood scales [35] A series of visual analogue scales were completed at the end of the cognitive assessment. Sixteen bipolar lines anchored at each end by an adjective describing a mood (e.g. tense/relaxed) were presented and participants selected a point on the scale that represented how they were feeling at that point in time. Individual item scores were calculated as \% distance along the line from the left. The individual scales were combined as recommended by the authors to form three mood factors: alert, calm, content.

\section{Procedure}

Participants were required to attend two 90-min sessions at the Brain, Performance and Nutrition Research Centre at Northumbria University, which took place between 1 and $3 \mathrm{pm}$ in the afternoon. Sessions were conducted between 6 and 7 days apart to ensure sufficient wash out between conditions. All testing took place in a suite of dedicated temperature-controlled university laboratories with participants visually isolated from each other and wearing noise-reduction headphones to decrease the impact of any auditory distractions. All participants were asked to consume the same breakfast and lunch on both study days, and were instructed to fast for at least $1 \mathrm{~h}$, and to abstain from caffeine for at least $2 \mathrm{~h}$, prior to testing. A food diary was utilised to establish compliance to these instructions. Occasional smokers were asked to abstain from smoking on the day of testing. Prior to arrival, participants were randomly allocated to one of two treatment orders as selected through a Latin square.

Following arrival at the laboratory, volunteers were initially briefed and provided written informed consent and their eligibility to participate was confirmed. Participants were then required to provide age and sex information and to complete a food diary. In order to establish baseline performance for that day, participants initially completed a pre-dose 20-min assessment of cognition and mood. This was followed immediately by ingestion of their juice for that day; 5-min was allowed for consumption of the juice through a straw. Following a 20-min absorption period, participants completed the post-dose assessment of cognition and mood. The second study visit was completed at the same time of day for each participant and was identical with the exception of the juice consumed.

\section{Statistics}

In order to minimise the number of comparisons, cognitive scores were collapsed into four composite scores as described above (accuracy for attention tasks, reaction time for attention tasks, accuracy for memory tasks, reaction time for memory tasks) and mood scales were combined to form three factors as recommended by the authors (calm, content, alert) [35].

To assess the possibility of any pre-treatment differences in cognitive performance or mood, baseline data were analysed with linear mixed models including the terms treatment, order, and treatment $\times$ order as fixed effects. Postdose data were then analysed with linear mixed models including the respective baseline as a covariate and the terms treatment, order, and treatment $\times$ order as fixed effects. Significant interactions were further explored with pairwise comparisons. Finally, in order to ascertain if any effects on cognition were related to effects on mood, and vice versa, a bivariate Pearson correlation was conducted on change in composite cognitive scores and change in mood ratings for each treatment condition. All data were analysed using SPSS version 22 for windows.

\section{Results}

Mean pre-dose baseline and post-dose scores for each treatment are presented in Table 3, along with standard deviations, $F$, and $p$ values. Data capture errors with two participants' data during the placebo arm and two participants' data during the purple grape arm resulted in only 16 datasets being analysed for attention measures.

\section{Baseline scores}

There were no statistically significant differences between treatments at baseline. However, there was a main effect of order on attention accuracy $[F(1,14)=4.82, p=0.046]$. This indicated that those who consumed placebo first performed better at baseline on both visits $(M=0.30$, $\mathrm{SE}=2.35)$, compared to those who received purple grape first $(M=-0.43, \mathrm{SE}=2.35)$. There was also a treatment $\times$ order interaction effect on memory reaction time $[F(18)=5.73, p=0.028]$. Pairwise comparisons revealed that whilst there was no significant difference between visits in those who consumed placebo first, those who received purple grape first performed better at baseline on visit $2(M=-0.30, \mathrm{SE}=0.29)$ than visit $1(M=0.25$, $\mathrm{SE}=0.29)(p=0.018)$.

\section{Post-dose scores}

\section{Attention RT}

A significant main effect of treatment $[F(1,13.54)=4.79$, $p=0.047]$ was evinced on the reaction time for attention tasks composite $z$-score. Examination of means revealed 
Table 3 Unadjusted baseline and post-dose mean and SD for composite $z$-scores and mood ratings

\begin{tabular}{|c|c|c|c|c|c|c|c|c|c|c|c|}
\hline \multirow[t]{2}{*}{ Measure } & \multirow[t]{2}{*}{ Treatment } & \multirow[t]{2}{*}{$N$} & \multicolumn{4}{|c|}{ Baseline } & \multicolumn{4}{|c|}{ Post-dose } & \\
\hline & & & Mean & SD & $F$ & $p$ & Mean & SD & $F$ & $p$ & \\
\hline \multirow[t]{3}{*}{ Memory accuracy $(\%)$} & Placebo & 20 & 0.27 & 0.5 & 1.74 & 0.204 & -0.26 & 0.59 & 2.03 & 0.173 & Treatment \\
\hline & Purple grape & 20 & 0.11 & 0.48 & 1.38 & 0.256 & -0.12 & 0.65 & 0.08 & 0.787 & Order \\
\hline & & & & & 0.07 & 0.787 & & & 1.69 & 0.212 & Treatment $\times$ order \\
\hline \multirow[t]{3}{*}{ Memory RT (ms) } & Placebo & 20 & 0.03 & 0.82 & 1.64 & 0.217 & -0.17 & 0.82 & 0.09 & 0.770 & Treatment \\
\hline & Purple grape & 20 & 0.22 & 1.02 & 0.65 & 0.431 & -0.09 & 0.70 & 0.04 & 0.841 & Order \\
\hline & & & & & 5.73 & 0.028 & & & 0.54 & 0.474 & Treatment $\times$ order \\
\hline \multirow[t]{3}{*}{ Attention accuracy $(\%)$} & Placebo & 16 & -0.04 & 0.85 & 0.11 & 0.744 & 0.08 & 0.69 & 0.56 & 0.472 & Treatment \\
\hline & Purple grape & 16 & -0.08 & 0.71 & 4.82 & 0.046 & 0.14 & 0.45 & 0.43 & 0.525 & Order \\
\hline & & & & & 0.54 & 0.473 & & & 0.10 & 0.763 & Treatment $\times$ order \\
\hline \multirow[t]{3}{*}{ Attention RT (ms) } & Placebo & 16 & -0.19 & 0.63 & 0.27 & 0.609 & 0.13 & 0.77 & 4.79 & 0.047 & Treatment \\
\hline & Purple grape & 16 & -0.13 & 0.66 & 0.30 & 0.593 & -0.18 & 0.87 & 0.87 & 0.367 & Order \\
\hline & & & & & 1.09 & 0.313 & & & 0.06 & 0.811 & Treatment $\times$ order \\
\hline \multirow[t]{3}{*}{ Bond-Lader Alert } & Placebo & 20 & 46.1 & 16 & 2.09 & 0.165 & 50.5 & 14.7 & 0.45 & 0.513 & Treatment \\
\hline & Purple grape & 20 & 52.6 & 11.1 & 0.53 & 0.474 & 50.9 & 14.2 & 0.02 & 0.893 & Order \\
\hline & & & & & 0.57 & 0.462 & & & 0.04 & 0.836 & Treatment $\times$ order \\
\hline \multirow[t]{3}{*}{ Bond-Lader Content } & Placebo & 20 & 61.7 & 14 & 0.80 & 0.382 & 60.3 & 13.3 & 0.12 & 0.737 & Treatment \\
\hline & Purple grape & 20 & 65 & 13.6 & 0.04 & 0.842 & 63.8 & 13.6 & 2.62 & 0.124 & Order \\
\hline & & & & & 0.53 & 0.476 & & & 1.59 & 0.223 & Treatment $\times$ order \\
\hline \multirow[t]{3}{*}{ Bond-Lader Calm } & Placebo & 20 & 64.2 & 18.2 & 1.23 & 0.281 & 58.7 & 12.6 & 4.57 & 0.046 & Treatment \\
\hline & Purple grape & 20 & 59.1 & 16.2 & 0.25 & 0.625 & 62.1 & 12.2 & 6.69 & 0.019 & Order \\
\hline & & & & & 0.67 & 0.425 & & & 1.62 & 0.220 & Treatment $\times$ order \\
\hline
\end{tabular}

Fig. 1 Adjusted means and SE for treatment effects on a attention RT and $\mathbf{b}$ calm ratings and for order effects on c calm ratings following placebo and purple grape juice $(R T$ reaction time; ${ }^{*} p<0.05$; order $1=$ placebo then purple grape, order $2=$ purple grape then placebo)
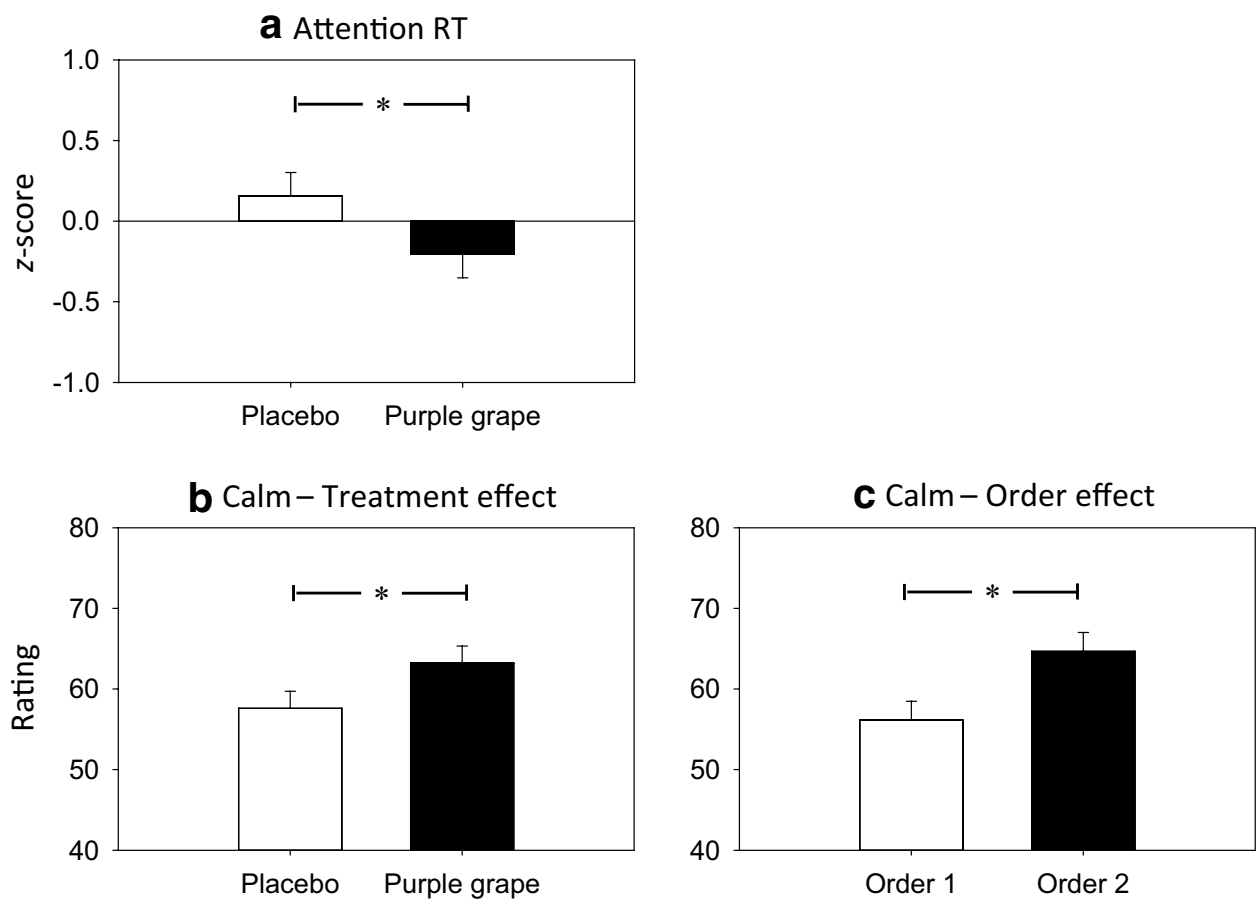
that participants were significantly faster following consumption of purple grape $(M=-0.21, \mathrm{SE}=0.15)$ relative to placebo $(M=0.16, \mathrm{SE}=0.15)$ (see Fig. 1a).

\section{Calm ratings}

A significant main effect of treatment on calm ratings $[F(1,18.37)=4.57, p=0.046]$ was found to be due to significantly higher calm ratings following consumption of purple grape $(M=63.22, \mathrm{SE}=2.10)$ compared to placebo $(M=57.61, \mathrm{SE}=2.10)$; see Fig. 1b. A significant main effect of order was also evinced $[F(1,17.82)=6.69$, $p=0.019]$, which indicated that post-dose calm ratings were significantly higher in those who received purple grape first $(M=64.68, \mathrm{SE}=2.33)$, than those who consumed placebo first $(M=56.15, \mathrm{SE}=2.33)$ (see Fig. 1c).

There were no other significant effects of treatment.

\section{Correlation between cognitive and mood scores}

No significant correlations between change in mood ratings and composite cognitive scores were observed.

\section{Discussion}

The results of the current study demonstrate for the first time, that acute consumption of purple grape juice can improve aspects of cognitive performance and mood in healthy young adults. Compared to the sugar-matched, low (poly)phenol control drink, purple grape juice enhanced overall speed on attention tasks and significantly increased calm ratings.

This is the first study to explore acute effects of purple grape on attention and adds to a growing body of evidence demonstrating improved attention task performance following acute supplementation with (poly)phenol-rich foods. Specifically, blackcurrant has been observed to decrease digit vigilance reaction time following a cold-pressed juice extract and to improve accuracy of rapid visual information processing (RVIP) following a freeze-dried extract in young healthy adults [26]. Similarly, cocoa consumption was shown to improve serial three subtraction performance in healthy young adults, with a dose-specific increase to speed of RVIP [25]. However, a recent 12-week intervention in 40- to 50-year-old mothers failed to find an effect of Concord grape juice (CGJ) on RVIP [23] raising the possibility that this effect is only seen in younger adults or is modified following repeated consumption. Recent data demonstrating improved continuous performance task accuracy and increased simple finger tapping following acute orange juice in middle-age males [27] would argue for the latter of these suggestions and this is supported by evidence for acute improvement to digit vigilance accuracy and serial three subtraction performance in elderly adults following curcumin consumption [36]. However, differences in methodology make direct comparisons difficult and further work is required exploring effects on attention in older adults, as this is often overlooked in favour of memory given its susceptibility to ageing. The lack of effects on memory in the current study is interesting as acute supplementation with wild blueberry has been shown to improve memory in 7to 10-year-old children [37], whereas the studies described above in middle-aged [27] and elderly participants [36] failed to find acute effects on memory. Taken together these findings may suggest that, in general, repeated consumption of (poly)phenols is required to observe improvements to memory, but that there are specific stages of development which are sensitive to acute manipulation.

A number of mechanisms have been proposed for the action of phenolic compounds on brain function; however, acute cognitive effects of (poly)phenols are commonly attributed to increased cerebral blood flow (CBF). Declines in CBF are observed in ageing [38, 39] and greater cerebral blood flow velocity has been related to significantly less cognitive decline [40], highlighting the importance of CBF modulation to cognition. Increases in $\mathrm{CBF}$ parameters have previously been demonstrated following supplementation with flavonoid-rich foods [41, 42], including increased activation in the right middle frontal and right superior parietal cortex following CGJ as measured with fMRI in mildly cognitively impaired adults [22]. The involvement of the fronto-parietal cortical networks in visual attention [43, 44] and the specific role for the right middle frontal and right superior parietal cortex in sustained attention [45, 46] suggest that this increase in activation may relate to the finding of increased speed of attention responses in the current study. However, the lack of attention measures employed by Krikorian et al. [22] and the wide-reaching involvement of the fronto-parietal cortex in other processes not affected here make it difficult to draw firm conclusions at this stage. Importantly, a study of cocoa flavan-3-ol supplementation in older adults, revealed significant benefits to cognition and cerebral blood volume (CBV) in the high flavan-3-ol group relative to control and greater $\mathrm{CBV}$ was correlated with better cognitive task performance [47]. Increases to CBF have also been observed in healthy young adults following (poly)phenols [48, 49], demonstrating that these modulations are not restricted to older populations who may be suffering declines in CBF.

One other explanation for the observed improvement to attention reaction time is a modulation of glucose metabolism. Berries have been shown to modulate the glucose and insulin response when compared to matched sucrose and available carbohydrate levels served in water $[50,51]$; and to improve the glycaemic profile of bread 
[52]. Grape seed extract co-consumed with a high-carbohydrate meal has also been shown to lower the post-prandial blood glucose response when measured at 15 and 30 -min post-consumption, as well as reducing the area under the curve for the 2-h period of measurement [53]. The impact of glucoregulation on cognition may depend upon the mechanism underlying this effect of (poly)phenols, a number of which have been suggested [16, 54]. However, studies have demonstrated that impaired glucose tolerance is associated with poorer cognition (see [55] for review) and modulation of insulin resistance has been shown to be a significant predictor of cognition following 12-weeks supplementation of cocoa flavan-3-ols, accounting for 17 and $40 \%$ change in a composite cognition score in healthy and MCI elderly, respectively. In light of this, further work is required examining the impact of acute glucoregulatory effects of (poly)phenols in relation to cognitive performance. Given the multifunctional nature of (poly)phenol effects it is likely that all of these mechanisms have a role to play and are also inter-related, with endothelial nitric oxide (NO) representing a key molecule in this relationship [56].

In addition to an improvement to attention reaction time, participants also rated themselves to be significantly calmer following purple grape when compared to placebo. Similar acute improvements have been shown in healthy young adults following epigallocatechin gallate (EGCG), evinced as increased calm and decreased stress ratings [57]. Studies showing alerting effects following cocoa [25], blackcurrant [26], and orange juice [27] provide further evidence for acute effects of (poly)phenols on subjective state. A number of mechanisms potentially underlie the demonstrated effect on calm ratings, including modulation of cortisol levels [58] and interaction with $\mathrm{GABA}_{\mathrm{A}}$ receptors [59]. Mood effects may also be mediated by suppression of monoamine oxidase-B (MAOB) activity, which was recently reported to be inhibited by $96 \%$ following acute administration of blackcurrant juice in healthy young adults [26]. Although this inhibition was not matched by modulation of calm ratings, anxiolytic effects have been reported following chronic administration of berries to rodents [60] and modulation of MAO activity has been proposed as a mechanism for antidepressant-like effects observed following various (poly)phenols in rats [61-63]. Interestingly, speed on a sustained attention task was also increased by the blackcurrant juice in the study by Watson et al. [26], suggesting a potential role for MAO inhibition in the effects on attention reaction time in the current study. Given the possibility for a common mechanism of action and the potential for cognitive and mood effects to impact on one another, correlations were conducted to assess any relationship between the two. No significant associations were observed, but this may reflect a lack of power to detect such effects.

A particularly interesting outcome from the current study is the impact of drink order on cognitive and mood outcomes both pre- and post-dose. A treatment $\times$ order interaction on speed of memory indicated that those who had received purple grape at visit 1 were significantly faster at baseline on visit 2 than on visit 1. Similarly, those who received purple grape first had higher post-dose calm ratings than those who received placebo first. These findings both suggest an enduring positive effect of purple grape that had carried over into the second visit. This suggestion is supportive of previous findings showing carryover effects on verbal recall, executive function and driving ability following a 12-week intervention with CJG [23], as well as higher alertness and concentration in those who consumed CGJ first. The demonstration of carryover effects following a single ingestion suggests that these are the result of learning effects rather than enduring changes to physiological mediators such as CBV or glucoregulation as a result of insufficient washout. Order effects are often not considered in studies of this type and should be included in future studies to verify their importance.

Previous studies of cognitive effects of purple grape in humans have employed CGJ supplied for the purpose of the research and consumed at levels far in excess of that which would conventionally be consumed (up to $621 \mathrm{ml} /$ day), making it difficult to convey a clear message to consumers regarding the required dose to achieve the desired effects. The current study demonstrates that a single serving of a commercially available purple grape juice is sufficient to achieve improvements to both cognition and mood and, together with previous findings of improvements to driving ability, help in communicating the health benefits of purple grape to consumers in a meaningful way. A potential problem with using a product 'off-the-shelf' relates to a lack of standardisation, but analysis of the purple grape juice employed, conducted in November 2013, demonstrated strikingly similar levels of anthocyanins and total phenolics to those previously published with the same product [9]. This analysis also served to validate the use of white grape juice as a 'placebo' (chosen on the basis of its similarity to purple grape juice in terms of mouth feel, taste and sugar content) as phenolic levels were shown to be very low and the addition of blackcurrant cordial was also shown to have minimal impact on these levels. Limitations of the current study relate to the absence of assessment of habitual (poly) phenol intake and the lack of standardisation of diet prior to testing. In keeping with other facets of the study, this lack of standardisation of diet served to provide an ecologically valid demonstration of effects of purple grape juice akin to that which would be expected when consumed in everyday living. Examination of participant's food diaries revealed 
good compliance with instructions to consume the same food and drink on study days and to abstain from food for $1 \mathrm{~h}$, and caffeine for $2 \mathrm{~h}$, prior to testing. The food consumed was generally of a low-(poly)phenol content, with $70 \%$ of participants reporting no fruit or vegetable intake and the maximum reported being 1 portion of fruit and 1 portion of vegetable. However, it is important that food intake is standardised prior to intervention in order to minimise interference caused by differing nutrient consumption between participants. It is also particularly important to record habitual (poly)phenol intake as this has the potential to impact on gut microbiota, which in turn impacts on absorption of (poly)phenols [64, 65]. For this reason, the stipulation for a beige diet in studies of health effects of (poly)phenols may underestimate any such effects due to reduced bio-efficacy induced by the diet. Similarly, if glucoregulation is found to be an important mediator in the effects of (poly)phenols on cognition, then this may explain null findings when an overnight fast is implemented.

In conclusion, our study demonstrates an acute enhancement of speed of attention and increased calm ratings following purple grape juice in healthy young adults, adding to a growing body of evidence for cognitive benefits of (poly)phenols. These findings require further examination as attention measures are often overlooked in favour of tasks assessing memory, and mood ratings are often difficult to replicate due to their subjective nature. The mechanisms underlying these effects also require further investigation and future studies should strive to include measures of cerebral blood flow, glucoregulation and MAO activity in conjunction with cognitive and mood measures in order to further elucidate their role.

\section{Compliance with ethical standards}

Conflict of interest On behalf of all authors, the corresponding author states that there is no conflict of interest.

Open Access This article is distributed under the terms of the Creative Commons Attribution 4.0 International License (http://creativecommons.org/licenses/by/4.0/), which permits unrestricted use, distribution, and reproduction in any medium, provided you give appropriate credit to the original author(s) and the source, provide a link to the Creative Commons license, and indicate if changes were made.

\section{References}

1. Manach C, Scalbert A, Morand C, Remesy C, Jimenez L (2004) Polyphenols: food sources and bioavailability. Am J Clin Nutr 79(5):727-747

2. Chun OK, Chung S-J, Claycombe KJ, Song WO (2008) Serum c-reactive protein concentrations are inversely associated with dietary flavonoid intake in US adults. J Nutr 138(4):753-760
3. Karlsen A, Retterstol L, Laake P, Paur I, Kjolsrud-Bohn S, Sandvik L, Blomhoff R (2007) Anthocyanins inhibit nuclear factor-kappa B activation in monocytes and reduce plasma concentrations of pro-inflammatory mediators in healthy adults. J Nutr 137(8):1951-1954

4. Wang X, Ouyang YY, Liu J, Zhao G (2014) Flavonoid intake and risk of CVD: a systematic review and meta-analysis of prospective cohort studies. Br J Nutr 111(1):1-11. doi:10.1017/s000711451300278x

5. Rossi M, Negri E, Talamini R, Bosetti C, Parpinel M, Gnagnarella P, Franceschi S, Dal Maso L, Montella M, Giacosa A, La Vecchia C (2006) Flavonoids and colorectal cancer in Italy. Cancer Epidemiol Biomark Prev 15(8):1555-1558. doi:10.1158/1055-9965.epi-06-0017

6. Reglodi D, Renaud J, Tamas A, Tizabi Y, Socías SB, DelBel E, Raisman-Vozari R (2015) Novel tactics for neuroprotection in Parkinson's disease: Role of antibiotics, polyphenols and neuropeptides. Prog Neurobiol. doi:10.1016/j. pneurobio.2015.10.004

7. Commenges D, Scotet V, Renaud S, Jacqmin-Gadda H, Barberger-Gateau P, Dartigues JF (2000) Intake of flavonoids and risk of dementia. Eur J Epidemiol 16(4):357-363. doi:10.102 3/a:1007614613771

8. Dai Q, Borenstein AR, Wu Y, Jackson JC, Larson EB (2006) Fruit and vegetable juices and Alzheimer's disease: the kame project. Am J Med 119(9):751-759. doi:10.1016/j.amjmed.2006.03.045

9. Mullen W, Marks SC, Crozier A (2007) Evaluation of phenolic compounds in commercial fruit juices and fruit drinks. J Agric Food Chem 55(8):3148-3157

10. Stalmach A, Edwards CA, Wightman JD, Crozier A (2011) Identification of (poly) phenolic compounds in concord grape juice and their metabolites in human plasma and urine after juice consumption. J Agric Food Chem 59(17):9512-9522

11. Lamport DJ, Dye L, Wightman JD, Lawton CL (2012) The effects of flavonoid and other polyphenol consumption on cognitive performance: a systematic research review of human experimental and epidemiological studies. Nutr Aging 1(1):5-25

12. Lamport DJ, Saunders C, Butler LT, Spencer JP (2014) Fruits, vegetables, $100 \%$ juices, and cognitive function. Nutr Rev 72(12):774-789. doi:10.1111/nure.12149

13. Gasperotti M, Passamonti S, Tramer F, Masuero D, Guella G, Mattivi F, Vrhovsek U (2015) Fate of microbial metabolites of dietary polyphenols in rats: is the brain their target destination? ACS Chem Neurosci 6(8):1341-1352. doi:10.1021/ acschemneuro.5b00051

14. Spencer JP, Vafeiadou K, Williams RJ, Vauzour D (2012) Neuroinflammation: modulation by flavonoids and mechanisms of action. Mol Aspects Med 33(1):83-97. doi:10.1016/j. mam.2011.10.016

15. Rendeiro C, Rhodes JS, Spencer JP (2015) The mechanisms of action of flavonoids in the brain: direct versus indirect effects. Neurochem Int 89:126-139. doi:10.1016/j.neuint.2015.08.002

16. Williamson G (2013) Possible effects of dietary polyphenols on sugar absorption and digestion. Mol Nutr Food Res 57(1):48-57. doi:10.1002/mnfr.201200511

17. Casadesus G, Shukitt-Hale B, Stellwagen HM, Zhu X, Lee H-G, Smith MA, Joseph JA (2004) Modulation of hippocampal plasticity and cognitive behavior by short-term blueberry supplementation in aged rats. Nutr Neurosci 7(5-6):309-316

18. Williams RJ, Spencer JP (2012) Flavonoids, cognition, and dementia: actions, mechanisms, and potential therapeutic utility for Alzheimer disease. Free Radic Biol Med 52(1):35-45. doi:10.1016/j.freeradbiomed.2011.09.010

19. Spencer JP (2009) The impact of flavonoids on memory: physiological and molecular considerations. Chem Soc Rev 38(4):1152-1161. doi:10.1039/b800422f 
20. Vauzour D, Vafeiadou K, Rodriguez-Mateos A, Rendeiro C, Spencer JP (2008) The neuroprotective potential of flavonoids: a multiplicity of effects. Genes Nutr 3(3-4):115-126. doi:10.1007/ s12263-008-0091-4

21. Krikorian R, Nash TA, Shidler MD, Shukitt-Hale B, Joseph JA (2010) Concord grape juice supplementation improves memory function in older adults with mild cognitive impairment. Br J Nutr 103(5):730

22. Krikorian R, Boespflug EL, Fleck DE, Stein AL, Wightman JD, Shidler MD, Sadat-Hossieny S (2012) Concord grape juice supplementation and neurocognitive function in human aging. $\mathrm{J}$ Agric Food Chem 60(23):5736-5742

23. Lamport DJ, Lawton CL, Merat N, Jamson H, Myrissa K, Hofman D, Chadwick HK, Quadt F, Wightman JD, Dye L (2016) Concord grape juice, cognitive function, and driving performance: a 12-wk, placebo-controlled, randomized crossover trial in mothers of preteen children. Am J Clin Nutr 103(3):775-783. doi:10.3945/ajen.115.114553

24. Hendrickson SJ, Mattes RD (2007) No acute effects of grape juice on appetite, implicit memory and mood. Food Nutr Res 52:183-188

25. Scholey AB, French SJ, Morris PJ, Kennedy DO, Milne AL, Haskell CF (2010) Consumption of cocoa flavanols results in acute improvements in mood and cognitive performance during sustained mental effort. J Psychopharmacol 24(10):1505-1514

26. Watson AW, Haskell-Ramsay CF, Kennedy DO, Cooney JM, Trower T, Scheepens A (2015) Acute supplementation with blackcurrant extracts modulates cognitive functioning and inhibits monoamine oxidase-B in healthy young adults. J Funct Foods 17:524-539. doi:10.1016/j.jff.2015.06.005

27. Alharbi MH, Lamport DJ, Dodd GF, Saunders C, Harkness L, Butler LT, Spencer JP (2016) Flavonoid-rich orange juice is associated with acute improvements in cognitive function in healthy middle-aged males. Eur J Nutr 55(6):2021-2029. doi:10.1007/ s00394-015-1016-9

28. Baune BT, Fuhr M, Air T, Hering C (2014) Neuropsychological functioning in adolescents and young adults with major depressive disorder-a review. Psychiatry Res 218(3):261-271. doi:10.1016/j.psychres.2014.04.052

29. Lee J, Durst RW, Wrolstad RE (2005) Determination of total monomeric anthocyanin pigment content of fruit juices, beverages, natural colorants, and wines by the $\mathrm{pH}$ differential method: collaborative study. J AOAC Int 88(5):1269-1278

30. Zhang Q, Zhang J, Shen J, Silva A, Dennis DA, Barrow CJ (2006) A simple 96-well microplate method for estimation of total polyphenol content in seaweeds. J Appl Phycol 18(3):445450. doi:10.1007/s10811-006-9048-4

31. Dodd FL, Kennedy DO, Riby LM, Haskell-Ramsay CF (2015) A double-blind, placebo-controlled study evaluating the effects of caffeine and L-theanine both alone and in combination on cerebral blood flow, cognition and mood. Psychopharmacology 232(14):2563-2576. doi:10.1007/s00213-015-3895-0

32. Kennedy DO, Jackson PA, Forster J, Khan J, Grothe T, Perrinjaquet-Moccetti T, Haskell-Ramsay CF (2015) Acute effects of a wild green-oat (Avena sativa) extract on cognitive function in middle-aged adults: a double-blind, placebo-controlled, within-subjects trial. Nutr Neurosci. doi:10.1080/10284 15x.2015.1101304

33. Stonehouse W, Conlon CA, Podd J, Hill SR, Minihane AM, Haskell C, Kennedy D (2013) DHA supplementation improved both memory and reaction time in healthy young adults: a randomized controlled trial. Am J Clin Nutr 97(5):1134-1143. doi:10.3945/ajen.112.053371

34. Galioto R, Spitznagel MB (2016) The effects of breakfast and breakfast composition on cognition in adults. Adv Nutr (Bethesda, Md) 7(3):576s-589s. doi:10.3945/an.115.010231
35. Bond A, Lader M (1974) The use of analogue scales in rating subjective feelings. Br J Med Psychol 47(3):211-218

36. Cox KH, Pipingas A, Scholey AB (2015) Investigation of the effects of solid lipid curcumin on cognition and mood in a healthy older population. J Psychopharmacol 29(5):642-651. doi: $10.1177 / 0269881114552744$

37. Whyte AR, Schafer G, Williams CM (2016) Cognitive effects following acute wild blueberry supplementation in 7- to 10-year-old children. Eur J Nutr 55(6):2151-2162. doi:10.1007/ s00394-015-1029-4

38. Girouard H (1985) Iadecola C (2006) Neurovascular coupling in the normal brain and in hypertension, stroke, and Alzheimer disease. J Appl Physiol 100(1):328-335. doi:10.1152/ japplphysiol.00966.2005

39. Iadecola C (2004) Neurovascular regulation in the normal brain and in Alzheimer's disease. Nat Rev Neurosci 5(5):347360. doi:10.1038/nrn1387

40. Ruitenberg A, den Heijer T, Bakker SL, van Swieten JC, Koudstaal PJ, Hofman A, Breteler MM (2005) Cerebral hypoperfusion and clinical onset of dementia: the Rotterdam study. Ann Neurol 57(6):789-794. doi:10.1002/ana.20493

41. Fisher ND, Sorond FA, Hollenberg NK (2006) Cocoa flavanols and brain perfusion. J Cardiovasc Pharmacol 47(Suppl 2):S210-S214

42. Lamport DJ, Pal D, Moutsiana C, Field DT, Williams CM, Spencer JP, Butler LT (2015) The effect of flavanol-rich cocoa on cerebral perfusion in healthy older adults during conscious resting state: a placebo controlled, crossover, acute trial. Psychopharmacology. doi:10.1007/s00213-015-3972-4

43. Corbetta M (1998) Frontoparietal cortical networks for directing attention and the eye to visual locations: identical, independent, or overlapping neural systems? Proc Natl Acad Sci USA 95(3):831-838

44. Naghavi HR, Nyberg L (2005) Common fronto-parietal activity in attention, memory, and consciousness: shared demands on integration? Conscious Cogn 14(2):390-425. doi:10.1016/j. concog.2004.10.003

45. Lewin JS, Friedman L, Wu D, Miller DA, Thompson LA, Klein SK, Wise AL, Hedera P, Buckley P, Meltzer H (1996) Cortical localization of human sustained attention: detection with functional MR using a visual vigilance paradigm. J Comput Assist Tomogr 20(5):695-701

46. Pardo JV, Fox PT, Raichle ME (1991) Localization of a human system for sustained attention by positron emission tomography. Nature 349(6304):61-64

47. Brickman AM, Khan UA, Provenzano FA, Yeung LK, Suzuki W, Schroeter H, Wall M, Sloan RP, Small SA (2014) Enhancing dentate gyrus function with dietary flavanols improves cognition in older adults. Nat Neurosci 17(12):1798-1803. doi: $10.1038 / \mathrm{nn} .3850$

48. Francis S, Head K, Morris P, Macdonald I (2006) The effect of flavanol-rich cocoa on the fMRI response to a cognitive task in healthy young people. J Cardiovasc Pharmacol 47:S215-S220

49. Kennedy DO, Wightman EL, Reay JL, Lietz G, Okello EJ, Wilde A, Haskell CF (2010) Effects of resveratrol on cerebral blood flow variables and cognitive performance in humans: a double-blind, placebo-controlled, crossover investigation. Am J Clin Nutr 91(6):1590-1597

50. Torronen R, Kolehmainen M, Sarkkinen E, Mykkanen H, Niskanen L (2012) Postprandial glucose, insulin, and free fatty acid responses to sucrose consumed with blackcurrants and lingonberries in healthy women. Am J Clin Nutr 96(3):527533. doi:10.3945/ajen.112.042184

51. Torronen R, Sarkkinen E, Niskanen T, Tapola N, Kilpi K, Niskanen L (2012) Postprandial glucose, insulin and glucagonlike peptide 1 responses to sucrose ingested with berries in 
healthy subjects. Br J Nutr 107(10):1445-1451. doi:10.1017/ s0007114511004557

52. Torronen R, Kolehmainen M, Sarkkinen E, Poutanen K, Mykkanen H, Niskanen L (2013) Berries reduce postprandial insulin responses to wheat and rye breads in healthy women. J Nutr 143(4):430-436. doi:10.3945/jn.112.169771

53. Sapwarobol S, Adisakwattana S, Changpeng S, Ratanawachirin W, Tanruttanawong K, Boonyarit W (2012) Postprandial blood glucose response to grape seed extract in healthy participants: a pilot study. Pharmacogn Mag 8(31):192-196. doi:10.4103/0973-1296.99283

54. Hanhineva K, Torronen R, Bondia-Pons I, Pekkinen J, Kolehmainen M, Mykkanen H, Poutanen K (2010) Impact of dietary polyphenols on carbohydrate metabolism. Int J Mol Sci 11(4):1365-1402. doi:10.3390/ijms11041365

55. Lamport DJ, Lawton CL, Mansfield MW, Dye L (2009) Impairments in glucose tolerance can have a negative impact on cognitive function: a systematic research review. Neurosci Biobehav Rev 33(3):394-413. doi:10.1016/j.neubiorev.2008.10.008

56. Katusic ZS, Austin SA (2014) Endothelial nitric oxide: protector of a healthy mind. Eur Heart J 35(14):888-894. doi:10.1093/ eurheartj/eht544

57. Scholey A, Downey LA, Ciorciari J, Pipingas A, Nolidin K, Finn M, Wines M, Catchlove S, Terrens A, Barlow E, Gordon L, Stough C (2012) Acute neurocognitive effects of epigallocatechin gallate (EGCG). Appetite 58(2):767-770. doi:10.1016/j. appet.2011.11.016

58. Steptoe A, Gibson EL, Vuononvirta R, Williams ED, Hamer M, Rycroft JA, Erusalimsky JD, Wardle J (2007) The effects of tea on psychophysiological stress responsivity and post-stress recovery: a randomised double-blind trial. Psychopharmacology 190(1):91. doi:10.1007/s00213-006-0620-z

59. Hanrahan JR, Chebib M, Johnston GA (2011) Flavonoid modulation of GABA(A) receptors. Br J Pharmacol 163(2):234-245. doi:10.1111/j.1476-5381.2011.01228.x

60. Barros D, Amaral OB, Izquierdo I, Geracitano L, do Carmo Bassols Raseira M, Henriques AT, Ramirez MR (2006) Behavioral and genoprotective effects of Vaccinium berries intake in mice. Pharmacology Biochemistry and Behavior 84(2):229-234

61. Xu Y, Li S, Chen R, Li G, Barish PA, You W, Chen L, Lin M, $\mathrm{Ku}$ B, Pan J (2010) Antidepressant-like effect of low molecular proanthocyanidin in mice: involvement of monoaminergic system. Pharmacol Biochem Behav 94(3):447-453

62. Xu Y, Wang Z, You W, Zhang X, Li S, Barish PA, Vernon MM, Du X, Li G, Pan J (2010) Antidepressant-like effect of trans-resveratrol: involvement of serotonin and noradrenaline system. Eur Neuropsychopharmacol 20(6):405-413

63. Zhen L, Zhu J, Zhao X, Huang W, An Y, Li S, Du X, Lin M, Wang Q, Xu Y (2012) The antidepressant-like effect of fisetin involves the serotonergic and noradrenergic system. Behav Brain Res 228(2):359-366

64. Valdes L, Cuervo A, Salazar N, Ruas-Madiedo P, Gueimonde M, Gonzalez S (2015) The relationship between phenolic compounds from diet and microbiota: impact on human health. Food Funct 6(8):2424-2439. doi:10.1039/c5fo00322a

65. Cardona F, Andres-Lacueva C, Tulipani S, Tinahones FJ, Queipo-Ortuno MI (2013) Benefits of polyphenols on gut microbiota and implications in human health. J Nutr Biochem 24(8):14151422. doi:10.1016/j.jnutbio.2013.05.001 